\title{
Computational fluid dynamics simulation of spray dryers: transient or steady state simulation?
}

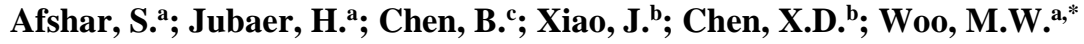

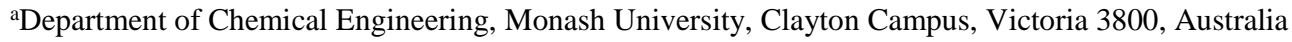 \\ ${ }^{\mathrm{b}}$ Chemical and Environmental Engineering, College of Chemistry, Chemical Engineering and Material \\ Sciences, Soochow University, Suzhou, Jiangsu, China \\ 'Department of Mechanical Engineering, Monash University, Clayton Campus, Victoria 3800, \\ Australia
}

*E-mail of the corresponding author: $\underline{\text { meng.woo@monash.edu }}$

\begin{abstract}
Self-sustained fluctuating airflow behaviour in spray drying chambers is in essence an unsteady phenomenon requiring the transient CFD simulation framework. There is currently, however, a mixture of steady state and transient CFD simulations of spray dryers practised and reported in the literature. The choice between steady state and transient approach significantly affects the computation time of the simulation and subsequently the adoption of this approach by industry. This paper firstly examines in detail the bottleneck in computation time of the transient simulation approach. Based on past reports, this review paper then presents a discussion and provides several recommendations on the use of steady state and transient simulation approach for spray dryers.
\end{abstract}

Keywords: CFD simulation, spray drying, transient, steady state, fluctuations 


\section{Introduction}

The start-up of an industrial spray drying operation typically involves an initial heating of the chamber with hot air, followed by the spray of water to mimic the evaporation rate of the intended product feed. Once the outlet air conditions are approximately achieved, the product feed spray is introduced. This is then followed by a short period of operation, during which the product is diverted and operation adjustments are made, until stable outlet air and product conditions are achieved. These stable conditions are called the steady state operating condition. Examining the overall spray drying process from such a macro plant-wide view, beyond the initial start-up phase, the a spray drying process is typically operated indeed in a steady state mode.

There are experimental evidences to suggest that even if the overall operation is steady state, the airflow pattern within the spray drying chamber may exhibit significant transient fluctuations [1][2]. Such transient air flow behaviour manifests in form of a self-sustained flapping from side to side. Such self-sustained transient behaviour was experimentally observed even for spray dryers fitted with nozzle and rotating disc atomizers (the latter would have significant swirl in the airflow pattern) and in large scale dryers with an internal static fluidized bed [3]. It is noteworthy that the self-sustained fluctuations were observed on relatively long time scales and were spatially coherent fluctuations; incontrast to small time scale and small length scale turbulent fluctuations.

Against the backdrop of a steady state spray drying operation, one may naturally raise the following question: Is it important for us to account for the self-sustained transient airflow behaviour in the analysis of spray dryers? Answering this question with a straightforward "yes" or "no" would naturally instigate a long debate and legitimate dispute, since it always boils down to the purpose of the simulation. However, if the interest is on the overall process i.e. mass and energy balance of the process, the answer is most likely "no". On the other hand, for specific studies such as those on detailed drying behaviour, wall deposition, agglomeration etc. particularly with the development in the application of the Computational Fluid Dynamics (CFD) technique for spray drying analysis, which currently allows detailed analysis of the flow field within the chamber to be performed, accounting for the selfsustained transient behaviour may become crucial. Reports available in the literature, however, are not conclusive on whether or not this phenomenon should be accounted for. The former, in essence, is unable to capture the self-sustained behaviour. This review paper provides a discussion and analysis based on these reports with the aim of providing a guideline for future works in selecting a suitable approach for CFD modelling of spray dryers.

\section{Steady versus transient CFD analysis and its implications}

It is important to firstly define the structure of a steady and a transient CFD analysis of spray dryer, so that the subsequent discussion can be made on the same benchmark. This review 
paper centres around the Euler-Lagrangian framework. Within this framework, the hot dehydrating air is computed in the Eulerian framework as a continuous medium while the particles are numerically injected into the simulated flow field as discrete Lagrangian phase. In this review, the term 'particles' refers to droplet or solid particles interchangeably. Twoway coupling is normally used in the simulation framework where the air imparts momentum, mass (moisture content) and energy change to the particles and vice versa; the latter two parameters are due to the drying process that the droplets are subjected to. Details on this aspects of a CFD simulation of spray dryers can be found in other publications [4].

Adopting the Euler-Lagrangian framework, Figure 1 shows two different numerical approaches in which a steady and a transient spray dryer simulation can be undertaken. Solutions with the transient framework will certainly involve higher computational requirements and longer computation time. From the authors' experience, however, given the computing power available nowadays, such differences may not be the significant factor affecting the potential selection between the steady and the transient framework. As a comparison, a steady state flow field simulation may take several hours while a transient flow field simulation may take a few days. The key difference lies in how the particles are injected into the flow field and in the way the two-way coupling is incorporated in both frameworks.

In the steady state framework, each particle is introduced into the flow field and tracked throughout the flow field until it leaves the simulation domain. This is then repeated depending on the number of particles specified to be injected to capture the stochastic behaviour of the particle movement and the particle size distribution of the spray. In essence, even though the source terms determined from the tracking of particles are accumulated for the subsequent incorporation into the steady state solution, there is only one particle numerically present in the simulation at any point. Therefore, the number of numerical loops required to achieve a converged solutions (Figure 1) is only dependent on the number of particle injection specified (which is fixed) and the 'numerical' speed at which the source terms are incorporated into the flow field solution. This 'numerical' speed is certainly dependent on the two-way coupling algorithm used and it is not the intention of this review paper to focus on any particular scheme.

Conversely, in the transient framework, particles are injected continuously at each discrete time step. During this time step, the particles injected into the simulation domain are only tracked and moved by one spatial step size. As opposed to steady state simulations, they are not tracked throughout the domain until those leave the chamber. Hence, as the simulation progresses in time, denoted by the loop in Figure 1, the number of particles within the chamber progressively increases leading to a further increase in the computational requirements at each time step. A fully developed flow field in this case, needs to account for not only the development of the temperature, momentum and humidity profile within the chamber, but also the development of the number of particles within the chamber. A well developed flow filed should have a relatively stable number of particles indicating that the system has 'truly' entered the steady state operation behaviour. The simulation time required to achieve such fully developed simulation is, therefore. significantly determined by the physical characteristics and the size of the spray dryer (larger drying chamber will expect to have more particle accumulation and vice versa) and not merely by the coupling algorithm, as in the steady state simulations. From the authors' experience, this takes significantly longer 
time, in the order of weeks for large scale industrial dryers, when compared to the steady state simulations.

The transient simulation framework also warrants significantly different approach to the postprocessing analysis of the particle drying history, which is one of the primary results from the CFD simulations. In the steady state approach, as each particle is tracked throughout the simulation domain until it leaves the domain, it is straightforward and easy to track and monitor the drying history of each particle. Determination of the particle conditions at the outlet of the dryer will only require collating the conditions of all the injected particle (those that leave the domain via the outlet) tracked one-by-one in the simulation. On the other hand, similar tracking and monitoring of the particle history in the transient framework will require large and simultaneous storage of all the particles within the simulation domain. For comparison, in a steady state simulation, an injection of hundreds or thousands of particles will normally be sufficient to account for the size distribution of the feed spray [5]. In a transient simulation, depending on the size of the dryer, the number of accumulated particles may be in the range of tens of thousands or hundreds of thousands of particles [6][7]. Determination of the particle conditions at the outlet will then require further time averaged sampling of particles leaving the system [6][7]. Even though the implemented parcel tracking method in the Eulerian-Lagrangian framework, which allows a parcel representing a group of identical particles having the same fate to be tracked in lieu of tracking each individual particle, eases the conundrum of tracking and storing information about so many particles, the remaining computational and postprocessing effort required still is far from negligible.

In order to overcome these limitations in transient simulation post-processing, a pseudosteady analysis method was developed in which the flow field is developed with fully transient airflow computation accompanied by transient particle tracking for a low velocity spray dryer. Particle history is then analysed and obtained by adopting an instantaneous snapshot of the developed flow field and undertaking steady state particle tracking through the flow field [8][9]. The premise of this post-processing approach assumes that, in view of the self sustained fluctuating behaviour, a snap shot at any particular flow time is representative of the overall flow behaviour in the system. Comparison on using snapshots at different flow time provided compelling evidence to support this assumption. Further comparison is, however, required with full transient analysis and with dryers of different configurations to better validate this technique. Even if this could be fully resolved or verified, the transient simulation framework would still need long computation time to fully develop the flow field incorporating transient simulation tracking (Figure 1). An earlier report can also be found adopting this approach excluding the two-way coupling [10] i.e. no buildup of humidity or reduction of the air temperature due to droplet evaporation could be predicted and fed back to the solution. 
Afshar, S.; Jubaer, H.; Chen, B.; Xiao, J.; Chen, X.D.; Woo, M.W.,

\section{Steady state framework}

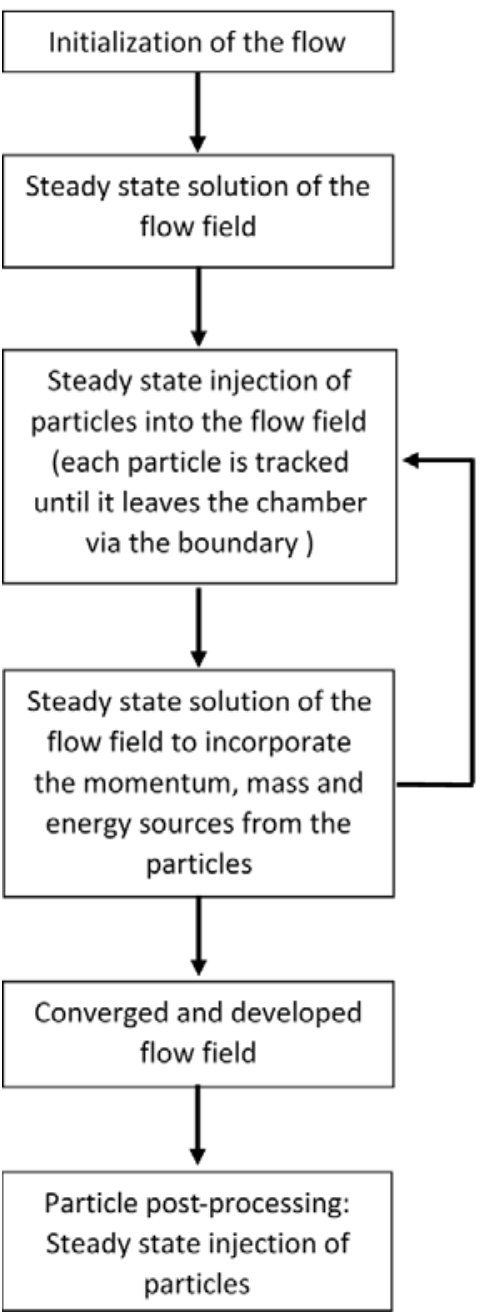

\section{Transient framework}

Initialization of the flow

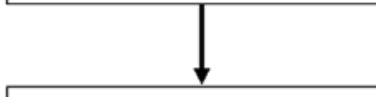

Pseudo-steady state solution of the flow field

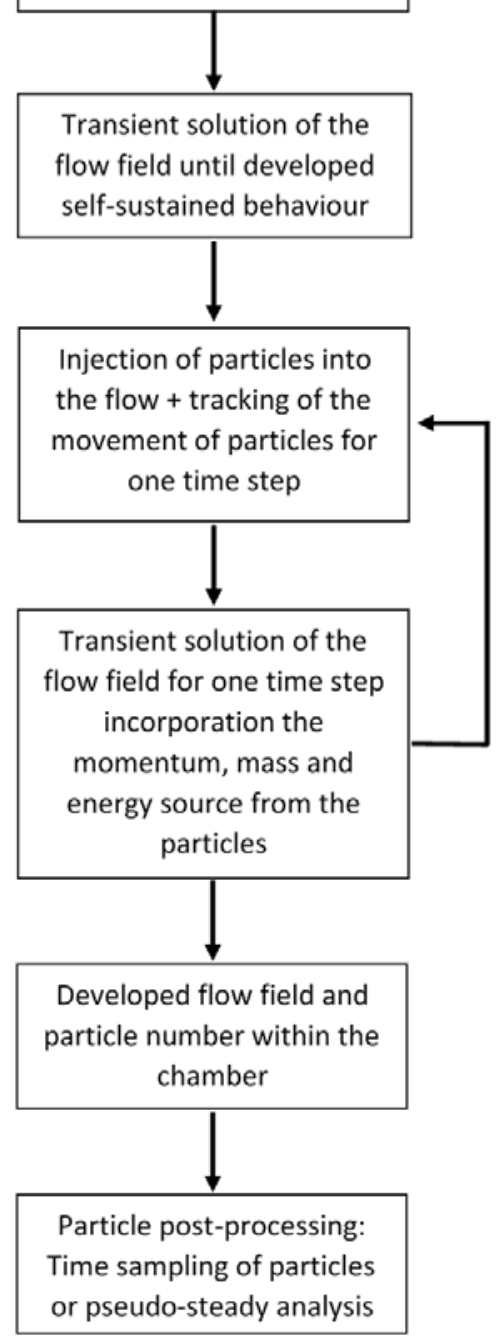

Fig. 1 Numerical approaches to undertaking steady or transient CFD simulations of spray dryers 
Based on the comparison presented above, it can be concluded that it is obviously more attractive to adopt the steady state simulation approach. This will then allow the CFD simulation technique to be used in a more routine-manner, which will be particularly important for the adoption of this technique by industry. Nevertheless, the same question still prevails: How accurately do steady state simulations capture the reality of a potentially fluctuating flow field in spray dryers? At any rate, the ensuing question will be: in what situation can we employ a steady state simulation? In an attempt to answer these important questions, detailed comparison were undertaken based on past reports in the literature. However, this part of the review was not included here due to page limitation.

\section{Understanding the numerical source of self-sustained fluctuation}

The fact that self-sustained fluctuations discussed in this review may occur even in symmetrical geometries suggests that it must be initiated from some 'imbalance' in the system. There is a series of numerically based reports elucidating the self-sustained fluctuation behaviour based on the sudden flow expansion theory. The premise of this theory is that a certain flow regime characterized by the Reynolds or Swirl number and the expansion ratio will have the propensity to exhibit self-sustained flow behavior [11][12][13][14]. The jet feedback mechanism was proposed later on, expanding on the theory of expansion to elucidate the potential unbalanced pressure within the chamber, leading to the self-sustained fluctuation behavior [15].

In a real physical system, there are many factors which may contribute to such imbalance such as slight asymmetry in the drying chamber or in the inlet flow of air (no perfect system in reality). These imbalances are then sustained or propagated and consequently reflected by the inherent propensity of the system for self-sustained fluctuations, as discussed in the preceding section. For a numerical system, however, where the geometry and the input parameters for a CFD simulation can be perfectly symmetrical, such asymmetric physical seed for the initiation of self-sustained fluctuation will not be available. One may argue that even though the geometry and the inputs are symmetrical, the meshing of the system, particularly if tetrahedral meshes are used, may lead to numerically asymmetric solution to the flow field. However, it must be noted that self-sustained fluctuations were predicted even when very structured symmetrical hexahedral meshes were used [14]. Basing our discussion on the solution of the turbulent flow field with the Reynolds Averaged Navier Stokes (RANS) equations and its subsequent closure models, there is no stochastic element in the mathematical framework. Hence, if the mesh used is symmetrical, the RANS framework should theoretically provide a symmetrical solution. What is then the source of asymmetry, which provides the seed for the occurrence of self-sustained fluctuation behaviour in a numerical model?

Eliminating the possible influence of the physical geometry, meshing and mathematical model as discussed, the seed for or the instigation of 'numerical' self-sustained fluctuation 
must then be attributed to numerical imbalance during the solution of the flow field. Such numerical imbalance may propagate into imbalances predicted in the physical flow field, building up the potential for self-sustained fluctuations. If this argument is to be further extended, it can be noted that perhaps, the numerical coupling with particles introduced into the simulation may also contribute to such instability, as was reported by Jubaer et al. [16]. Whether or not such imbalances propagate into self-sustained flow behaviour will certainly depend on the propensity of the spray drying chamber for 'dampening' such physical or numerical imbalances. The understanding on the numerical source of self-sustained fluctuation is important in guiding the numerical approach employed in the CFD simulation of spray dryers. Perhaps, as a 'robust' approach to encompass all the possibilities to capturing the self-sustained fluctuation, there may be a need for an element of numerical imbalance in the simulation domain; a system which inherently is not self-sustained in fluctuation would damp the imbalance anyway and vice versa.

\section{Conclusions}

A key numerical bottleneck in transient CFD simulation of self-sustained fluctuating flows in spray dryers is the long computation time required for two-way coupling during flow development. There is a strong need for future numerical development to overcome this limitation so that the CFD technique can be used in a more routine manner. From the reports available in the literature, there is currently no clear guidelines on the use of steady or transient simulation approach when analysing pilot or industrial scale spray dryers. This paper recommends to ascertain the significance of the fluctuating behaviour with the transient simulation framework, on a case-by-case basis. This is particularly important if an asymmetric flow field was predicted for a symmetrical simulation domain. A few numerical guidelines are proposed. It was further reasoned that numerical imbalance may be the seed for self-sustained fluctuations observed in CFD simulations of spray dryers. Whether or not this numerical imbalance propagates into physical imbalances leading to flow fluctuation behaviours, depends on the characteristics of the spray drying geometry relative to its operating conditions, which may or may not contribute to dampening these imbalances.

\section{References}

[1] Southwell, D.B.; Langrish, T.A.G. The effect of swirl on flow stability in spray dryers. Chemical Engineering Research and Design 2001, 79: 222-234.

[2] Langrish, T.A.G.; Oakley, D.E.; Keey, R.B.; Bahu, R.E.; Hutchinson, C.A. Timedependent flow patterns in spray dryers. Chemical Engineering Research and Design 1993, 71: 355-360. 
[3] Gabites, J.R.; Abrahamson, J.; Winchester, J.A. Air flow patterns in an industrial milk powder spray dryer. Chemical Engineering Research and Design 2010, 88: 899-910.

[4] Woo, M.W. Computational Fluid Dynamic Simulation of Spray Dyers - An Engineer's Guide. 2016. CRC Press. US, Florida.

[5] Woo, M.W.; Daud, W.R.W.; Mujumdar, A.S.; Wu, Z.H.; Talib, M.Z.M.; Tasirin, S.M. CFD evaluation of droplet drying models in a spray dryer fitted with a rotary atomizer. Drying Technology 2008, 26(10), 1180-1198.

[6] Jin, Y.; Chen, X.D. A three-dimensional numerical study of the gas/particle interactions in an industrial-scale spray dryer for milk powder production, Drying Technology 2009, 27(10), 1018-1027

[7] Jin, Y.; Chen, X.D. Numerical study of the drying process of different sized particles in an industrial scale spray dryer. Drying Technology 2009, 27(3), 371-381.

[8] Woo, M.W.; Rogers, S.; Lin, S.X.Q.; Selomulya, C.; Chen, X.D. Numerical probing of a low velocity concurrent pilot scale spray drying tower for mono-disperse particle production - unusual characteristics and possible improvements. Chemical Engineering and Processing 2011, 50(4), 417-427.

[9] Woo, M.W.; Rogers, S.; Selomulya, C.; Chen, X.D. Particle drying and crystallization characteristics in a low velocity concurrent pilot scale spray drying tower. Powder Technology 2011, 223, 39-45.

[10] Kota, K.; Langrish, T.A.G. Prediction of Deposition Patterns in a Pilot-Scale Spray Dryer Using Computational Fluid Dynamics (CFD) Simulations. Chemical Product and Process Modeling 2007, 2(3): Article 26.

[11] Fletcher D.F.; Guo B.; Harvie D.J.E.; Langrish T.A.G.; Nijdam J.J.; Williams J. What is important in the simulation of spray dryer performance and how do current CFD models perform? Applied Mathematical Modelling 2006, 30, 1281-1292.

[12] Guo, B.; Langrish, T.A.G.; Fletcher, D.F. CFD simulation of precession in sudden pipe expansion flows with low inlet swirl. Applied Mathematical Modelling 2002, 26: 1-15.

[13] Guo, B.; Langrish, T.A.G.; Fletcher, D.F. Simulation of gas flow instability in a spray dryer. Chemical Engineering Research and Design 2003, 81(A): 631-638.

[14] Guo, B.; Langrish, T.A.G.; Fletcher, D.F. Simulation of turbulent swirl flow in an axisymetric sudden expansion, AIAA Journal 2001, 39(1): 96-102.

[15] Woo, M.W.; Daud, W.R.W.; Mujumdar, A.S.; Talib, M.Z.M.; Wu, Z.H.; Tasirin, S.M. Non-swirling steady and transient flow simulations in short-form spray dryers. Chemical Product and Process Modelling 2009, 4(1), 20.

[16] Jubaer, H.; Afshar, S.; Xiao, J.; Chen, X.D.; Selomulya, C.; Woo, M.W. On the Importance of Droplet Shrinkage in CFD-Modelling of Spray Drying. Drying Technology 2017 (doi: 10.1080/07373937.2017.1349791) 